\title{
ÁRBOLES DE FORZAMIENTO SEMÁNTICO PARA LA SEMÁNTICA DE SOCIEDADES ABIERTAS
}

\section{SEMANTIC FORCING TREES FOR SEMANTICS OF OPEN SOCIETIES}

\author{
Manuel SIERRA-ARISTIZÁBAL \\ Departamento de Ciencias Matemáticas, Universidad Eafit, Medellín, Colombia. \\ * Autor corresponsal. E-mail: mahesiar@outlook.com
}

Historia del artículo

Recibido: Abril 10, 2018

Evaluado: Agosto 3, 2018

Aceptado: Agosto 13, 2018

Disponible: Septiembre 19, 2018

\section{Resumen}

La semántica de sociedades biasertivas abiertas para el sistema de lógica paraconsistente P1 se caracteriza por una herramienta de inferencia visual denominada "árboles de forzamiento semántico para sociedades abiertas". Dada una fórmula, con esta herramienta se marcan los nodos del árbol asociado a esta y se determina si la fórmula es válida o no. En el caso en que la fórmula sea inválida la sociedad que la refuta está determinada por las marquillas de las hojas en su árbol de forzamiento.

Palabras clave: árbol de forzamiento; semántica; sociedad abierta

\section{Abstract}

The semantics of open biassertive societies for the paraconsistent logic system P1, is characterized by a visual inference tool called semantic forcing trees for open societies. Given a formula, with this tool the nodes of the corresponding tree are marked, and it is determined whether the formula is valid or not. In case the formula is invalid, the society that refutes it is determined by the leaf markers in its forcing tree.

Keywords: forcing tree; semantics; biassertive societies

\section{INTRODUCCIÓN}

El método de las tablas semánticas se presenta en Beth (1962), y lo populariza Smullyan (1968) con el nombre de "árboles de opciones semánticas". Con este método se examinan de forma sistemática todas las opciones que pueden refutar una proposición dada, y se determina si una de estas opciones es lógicamente viable al no generar contradicciones. Si esto ocurre, se tiene un contraejemplo, con el cual se refuta la validez de la proposición dada. Si es imposible generar el contraejemplo, es decir, si ninguna de las opciones resulta lógicamente posible, entonces la proposición analizada es válida. Este método ha tenido amplia aceptación, y como lo han hecho Fitting (1971), Carnielli (1987), o Barrero y Carnielli (2005), se ha aplicado a muchos sistemas de lógicas no clásicas.
Los árboles de forzamiento semántico que presenta Sierra (2001; 2006) no buscan construir el contraejemplo al explorar todas las opciones posibles (tal como se realiza con las tablas semánticas), sino que los árboles de forzamiento solo consideran las opciones que son deductivamente forzadas por las reglas del sistema. En consecuencia, el análisis de validez con los árboles de forzamiento semántico es más sencillo y natural con relación al que se realiza con los árboles de opciones.

La semántica de sociedades biasertivas abiertas la presentan Carnielli y Lima-Marques (1999) a fin de caracterizar el sistema deductivo $\mathrm{P}^{1}$ presentado en Sette (1973). El sistema $\mathrm{P}^{1}$ es paraconsistente y soporta las contradicciones a nivel de fórmulas atómicas. En las semánticas de sociedades el valor de 
verdad de la negación de una fórmula atómica no depende del valor de verdad de la fórmula atómica; los valores de verdad de este par de fórmulas dependen de la existencia de agentes en la sociedad que las validen o que las refuten. Es importante resaltar que el enfoque de la semántica de sociedades, a fin de solucionar el problema de las inconsistencias a nivel atómico, también es de interés para investigadores de otras áreas de la ciencia, tales como los sistemas basados en conocimiento (por ejemplo, las inconsistencias pueden aparecer en las bases de datos debido a la incompleta descripción de la información o a la descripción conflictiva generada por las diversas fuentes).

En Guarín y Montoya (2003) se extienden los árboles de forzamiento semántico para el cálculo proposicional clásico con reglas que permiten generar sociedades abiertas, con el fin de refutar fórmulas inválidas del sistema deductivo paraconsistente LBPCAt presentado en Sierra (2003).

\section{MATERIALES Y MÉTODOS}

En este trabajo se presentan los árboles de forzamiento semántico para sociedades abiertas. Se prueba con todo detalle la equivalencia entre la presentación con árboles de forzamiento y la presentación semántica de las sociedades abiertas. Finalmente, se muestra que, si una fórmula es inválida - lo cual implica que el árbol de forzamiento de la fórmula está bien marcado-, entonces la lectura de las marquillas de las fórmulas cuasiatómicas proporciona una sociedad biasertiva abierta, con la cual se refuta la validez de la fórmula.

\section{RESULTADOS Y DISCUSIÓN |}

\section{Lenguaje de la lógica de las sociedades biasertivas abiertas (LSA)}

El lenguaje de LSA consta de los conectivos binarios: $\rightarrow, \wedge$, $\vee$ y $\leftrightarrow$ (condicional, conjunción, disyunción y bicondicional); de los conectivos monádicos: I, $\sim$ y $\neg$ (incompatibilidad, negación y cuestionamiento); además, del paréntesis izquierdo y el paréntesis derecho. También se tiene una cantidad enumerable de fórmulas atómicas. El conjunto de fórmulas de LSA lo generan las siguientes reglas y solo ellas:

- Toda fórmula atómica es una fórmula.

- $\quad$ Si $X$ es una fórmula entonces $\sim(X)$ y $\neg(X)$ son fórmulas.

- Si p es una fórmula atómica entonces Ip es una fórmula.

- Si p es una fórmula atómica entonces $\neg p$ y p son fórmulas cuasiatómicas.

- $\quad$ Si X y Y son fórmulas entonces $(X) \wedge(Y),(X) \vee(Y),(X) \rightarrow(Y)$ $y(X) \leftrightarrow(Y)$ son fórmulas.

\section{Árbol de una fórmula}

Sea $X$ una fórmula, el árbol inicial de $X$ se representa por $\operatorname{Ar}[X]$ y se construye al utilizar las reglas presentadas en la (Fig. 1), donde A y B son fórmulas arbitrarias.

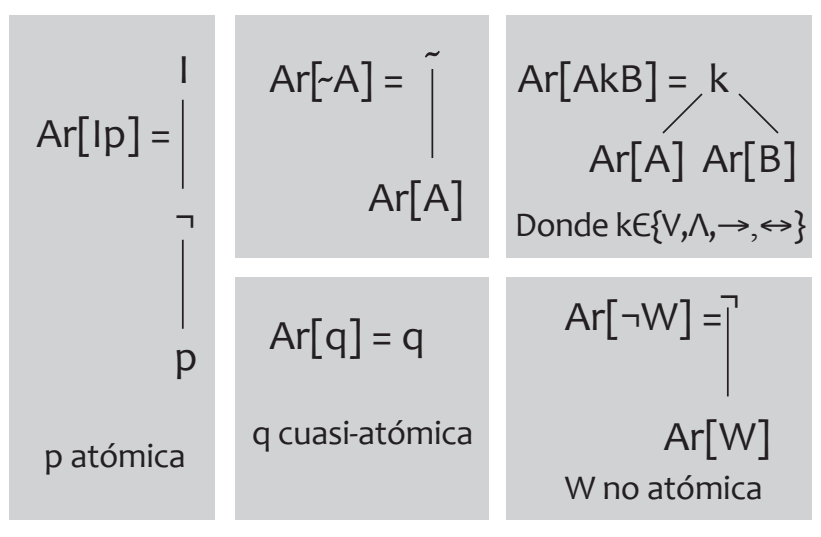

Figura 1.

Se define el árbol inicial del argumento 'de $X_{1}, \ldots, X_{n}$ se infiere $Y^{\prime}$, como: $\operatorname{Ar}\left[\left(X_{1} \wedge \ldots \wedge X_{n}\right) \rightarrow Y\right]$. El nodo superior del árbol de la fórmula $X$ se denomina "la raíz del árbol", se denota $\mathrm{R}[\mathrm{X}]$ y corresponde al operador principal de la fórmula $\mathrm{X}$. Los nodos inferiores, es decir, aquellos de los cuales no salen ramas, se denomina "hojas" y corresponden a las fórmulas cuasiatómicas.

Por ejemplo, para el argumento 'de $\neg A \rightarrow B$ y $\sim B \leftrightarrow I C$ se infiere $\neg[C \vee(A \wedge D)]$ ', donde $A, B, C$ y $D$ son fórmulas atómicas, el condicional asociado es: $[(\neg A \rightarrow B) \wedge(\sim B \leftrightarrow I C)] \rightarrow$ $\neg[C \vee(A \wedge D)]$ y su árbol inicial se presenta en la (Fig. 2$)$.

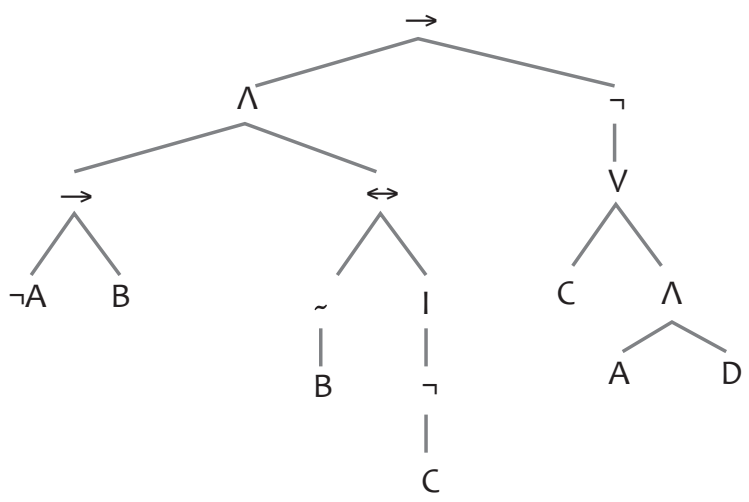

Figura 2.

\section{Marcando los nodos de un árbol}

Si un nodo $C$ es uno de los conectivos monádicos, I, $\sim 0 \neg$, entonces su único hijo se denomina "el alcance del operador", y a fin de hacer referencia a él se utiliza la notación $a C$.

Si un nodo $K$ es uno de los conectivos binarios $\wedge, \vee, \rightarrow 0$ $\leftrightarrow$, entonces para sus hijos izquierdo y derecho se utiliza la notación iK y $d K$, respectivamente.

Para toda fórmula $Y$, el nodo asociado a $Y$ es la raíz de $Y$, $R[Y]$, la cual a su vez es el operador principal de $Y$ en el caso 
que $Y$ no sea cuasiatómica, o es la misma $Y$ en el caso que $Y$ sea cuasiatómica.

Para una fórmula $\mathrm{X}, \mathrm{H}(\mathrm{X})$ el conjunto de hojas del $\operatorname{Ar}[\mathrm{X}]$, y $\mathrm{N}(\mathrm{X})$ el conjunto de nodos de $\operatorname{Ar}[\mathrm{X}]$.

Para cada fórmula $X$, una función de marca de hojas $m$ (o simplemente función de marca), es una función de $H(X)$ en $\{0,1\}$.

Si $m(p)=1$, entonces se dice que la hoja $p$ está marcada con 1 , o que es aceptada.

Si $m(p)=$ ó entonces se dice que la hoja $p$ está marcada con 0 , o que es rechazada.

Cada función de marca $m$ se encuentra determinada por un conjunto no vacío y enumerable de marquillas $\left\{m_{1}, m_{2}, \ldots\right.$, $\left.m_{n}, \ldots\right\}$, las cuales son funciones de $H(X)$ en $\{0,1\}$, definidas por las siguientes reglas:

- Aat. Aceptación de atómico: una fórmula atómica es aceptada si y solo si existe al menos una marquilla de la función de marca que la acepta.

$$
\mathrm{m}(\mathrm{at})=1 \Leftrightarrow \exists \mathrm{m}_{\mathrm{q}} \in \mathrm{m}, \mathrm{m}_{\mathrm{q}}(\mathrm{at})=1 \text { (1) }
$$

- A $\neg$ at. Aceptación del cuestionamiento de atómico: el cuestionamiento de una fórmula atómica es aceptado si y solo si existe al menos una marquilla de la función de marca que la rechaza.

$$
\mathrm{m}(\neg \mathrm{at})=1 \Leftrightarrow \exists \mathrm{m}_{\mathrm{q}} \in \mathrm{m}, \mathrm{m}_{\mathrm{q}}(\mathrm{at})=0 \text { (2) }
$$

Cada función de marca de hojas $m$ se extiende a una función de marca de nodos $M$ de $N(A)$ en $\{0,1\}$ según las reglas primitivas para el forzamiento de marcas que se presentan a continuación (las reglas primitivas para los operadores $\wedge, v$, $\rightarrow, \leftrightarrow \mathrm{y} \sim$ son las presentadas en Sierra (2006)).

\section{Reglas para marcar los nodos}

$M(h)=m(h)$ si h es una hoja.

- A $\quad$ nnat. Aceptación del cuestionamiento de no atómico: una fórmula no atómica es rechazada cuando es cuestionada.

$$
\mathrm{M}(\neg)=1 \Rightarrow \mathrm{M}(\mathrm{a} \neg)=0 \text { (3) }
$$

- Ra $\neg$ nat. Rechazo del alcance del cuestionamiento: las fórmulas no atómicas rechazadas son cuestionadas.

$$
M(a \neg)=0 \Rightarrow M(\neg)=1(4)
$$

- A Alat. Aceptación del cuestionamiento y aceptación de la incompatibilidad a nivel atómico: los enunciados atómicos cuestionados que son incompatibles con su cuestionamiento son rechazados.

$$
M(\neg)=M(I)=1 \Rightarrow M(a \neg)=0 \text { (5) }
$$

- Rlat. Rechazo de la incompatibilidad a nivel atómico: los enunciados atómicos compatibles con su cuestionamiento se aceptan y se cuestionan.

$$
M(I)=0 \Rightarrow M(\neg)=M(a \neg)=1(6)
$$

- $\quad A \neg A a \neg l a t$. Aceptación del cuestionamiento y aceptación del alcance del cuestionamiento en la incompatibilidad a nivel atómico: los enunciados atómicos que son aceptados y cuestionados son compatibles con su cuestionamiento.

$$
M(\neg)=M(a \neg)=1 \Rightarrow M(I)=0(7)
$$

Se dice que una fórmula $X$ es $A$-válida (válida desde el punto de vista de los árboles) si y solo si para toda función de marca $m$, se tiene que $M(R[X])=1$.

Se dice que una fórmula $X$ es $A$-inválida si no es $A$-válida, es decir, si existe una función de marca $m$, tal que $M(R[X])=0$. En este caso, se dice que la función de marca refuta la fórmula $X$. También se dice que el árbol de $X$ está bien marcado ( $A B M$, todos sus nodos están marcados de acuerdo con las reglas sin generar contradicciones).

\section{Reglas derivadas para el forzamiento de marcas}

Las reglas primitivas para el forzamiento de marcas son suficientes para estudiar las propiedades de los árboles de forzamiento. Sin embargo, en la práctica, cuando se trata de marcar todos los nodos de un árbol, es importante tener reglas que cubran todas las posibilidades. A continuación, se presenta un juego completo de reglas derivadas. Estas reglas derivadas para los conectivos $\wedge, v, \rightarrow, \leftrightarrow \mathrm{y} \sim$ se encuentran en Sierra (2006).

\section{Proposición. Reglas derivadas para el cuestionamiento}

- Aaᄀnat. Aceptación del alcance no atómico del cuestionamiento: las fórmulas no atómicas que son aceptadas no son cuestionadas.

$$
\mathrm{M}(\mathrm{a} \neg)=1 \Rightarrow \mathrm{M}(\neg)=0
$$

- $\quad R \neg n a t$. Rechazo del cuestionamiento de no atómico: si una fórmula no atómica no es cuestionada, entonces es aceptada.

$$
\mathrm{M}(\neg)=0 \Rightarrow M(\mathrm{a} \neg)=1
$$

- Rat. Rechazo de atómico: una fórmula atómica es rechazada, si y solo si todas las marquillas de la función de marca la rechazan.

$$
\mathrm{m}(\mathrm{at})=0 \Leftrightarrow \forall \mathrm{m}_{\mathrm{q}} \in \mathrm{m}, \mathrm{m}_{\mathrm{q}}(\mathrm{at})=0
$$

- $\quad \mathrm{R} \neg$ at. Rechazo del cuestionamiento de atómico: una fórmula atómica no es cuestionada, si y solo si todas las marquillas de la función de marca la aceptan.

$$
\mathrm{m}(\neg \mathrm{at})=0 \Leftrightarrow \forall \mathrm{m}_{\mathrm{q}} \in \mathrm{m}, \mathrm{m}_{\mathrm{q}}(\mathrm{at})=1
$$

- Prueba de $A a \neg$ nat: sea $M(a \neg)=1$. Supóngase que $M(\neg)=$ 1 , entonces por $A \neg$ nat se infiere $M(a \neg)=0$, lo cual no es el caso. Por tanto, forzosamente $\mathrm{M}(\neg)=0$.

- Prueba de $R \neg$ nat: sea $M(\neg)=0$. Supóngase que $M(a \neg)=$ 0 , entonces por $R a \neg$ nat se infiere $M(\neg)=1$, lo cual no es el caso. Por tanto, forzosamente $M(a \neg)=1$. 
- Prueba de Rat: por Aat se tiene que $\mathrm{m}(\mathrm{at})=1 \Leftrightarrow \exists \mathrm{m}_{\mathrm{q}} \in \mathrm{m}$, $\mathrm{m}_{\mathrm{q}}(\mathrm{at})=1$, lo cual implica que $\mathrm{m}(\mathrm{at})=0 \Leftrightarrow$ no $\exists \mathrm{m}_{\mathrm{q}}^{\mathrm{q}} \in \mathrm{m}$, $\mathrm{m}_{\mathrm{q}}(\mathrm{at})=1$, es decir, $\mathrm{m}(\mathrm{at})=0 \Leftrightarrow \forall \mathrm{m}_{\mathrm{q}} \in \mathrm{m}, \mathrm{m}_{\mathrm{q}}(\mathrm{at})=0$.

- $\quad$ Prueba de $\mathrm{R} \neg$ at: por $\mathrm{A} \neg$ at se tiene que $\mathrm{m}(\neg \mathrm{at})=1 \Leftrightarrow$ $\exists \mathrm{m}_{\mathrm{q}} \in \mathrm{m}, \mathrm{m}_{\mathrm{q}}(\mathrm{at})=0$, lo cual implica que $\mathrm{m}(\neg \mathrm{at})=0 \Leftrightarrow$ no $\exists \mathrm{m}_{\mathrm{q}} \in \mathrm{m}, \mathrm{m}_{\mathrm{q}}(\mathrm{at})=0$, es decir, $\mathrm{m}(\neg \mathrm{at})=0 \Leftrightarrow \forall \mathrm{m}_{\mathrm{q}} \in \mathrm{m}$, $m_{\mathrm{q}}(\mathrm{at})=1$.

\section{Proposición. Reglas derivadas para la incompatibilidad}

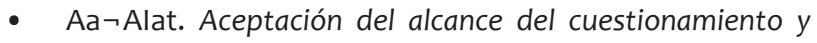
aceptación de la incompatibilidad a nivel atómico: no son cuestionadas las fórmulas atómicas que se aceptan y además sean incompatibles con su cuestionamiento.

$$
M(\mathrm{a} \neg)=\mathrm{M}(\mathrm{I})=1 \Rightarrow \mathrm{M}(\neg)=0
$$

- $\quad \mathrm{R} \neg$ lat. Rechazo del cuestionamiento en la incompatibilidad a nivel atómico: si una fórmula atómica no es cuestionada entonces es incompatible con su cuestionamiento.

$$
\mathrm{M}(\neg)=0 \Rightarrow \mathrm{M}(\mathrm{I})=1
$$

- Raᄀlat. Rechazo del alcance del cuestionamiento en la incompatibilidad a nivel atómico: si una fórmula atómica es rechazada entonces es incompatible con su cuestionamiento.

$$
M(a \neg)=0 \Rightarrow M(I)=1
$$

- $\quad$ Prueba de $A a \neg A l a t:$ sean $M(a \neg)=M(I)=1$. Si $M(\neg)=1$, como $M(I)=1$, por $A \neg$ Alat se infiere que $M(a \neg)=0$, lo cual no es el caso.

- $\quad$ Prueba de $R \neg$ lat: sea $M(\neg)=0$. Si $M(I)=0$, por Rlat se deriva que $M(\neg)=1$, lo cual no es el caso.

- Prueba de $R a \neg l a t:$ sea $M(a \neg)=0$. Si $M(I)=0$, por Rlat se deriva que $M(a \neg)=1$, lo cual no es el caso.

\section{Semántica para la lógica de sociedades abiertas}

De acuerdo con Carnielli y Lima-Marques (1999), y Guarín y Montoya (2003), una sociedad abierta s consta de un conjunto enumerable de agentes $\left\{\mathrm{Ag}_{1}, \mathrm{Ag}_{2}, \ldots, \mathrm{Ag}_{\mathrm{n}}, \ldots\right\}$. Cada agente es una función que interpreta las fórmulas cuasiatómicas de LSA $\{p, \neg p: p$ atómica $\}$ como elementos del conjunto $\{0,1\}$. La sociedad abierta $s$ se extiende a una función $S$ que interpreta las fórmulas de LSA en el conjunto $\{0,1\}$, mediante las siguientes reglas primitivas para las sociedades abiertas:

- $\quad$ sat. $s(p)=1 \Leftrightarrow \exists A g \in s, A g(p)=1$, donde $p$ es una fórmula atómica.

- $\quad s \neg$ at. $s(\neg p)=1 \Leftrightarrow \exists A g \in s, A g(p)=0$, donde $p$ es una fórmula atómica.

- Scuasi. Si c es fórmula cuasi-atómica entonces $S(c)=s(c)$.

- $\quad S \sim . S(\sim X)=1 \Leftrightarrow S(X)=0$.

- $S \wedge . S(X \wedge Y)=1 \Leftrightarrow S(X)=S(Y)=1$.

- $\quad S \vee S(X \vee Y)=0 \Leftrightarrow S(X)=S(Y)=0$.

- $\mathrm{S} \rightarrow . \mathrm{S}(\mathrm{X} \rightarrow \mathrm{Y})=0 \Leftrightarrow \mathrm{S}(\mathrm{X})=1$ y $\mathrm{S}(\mathrm{Y})=0$.
- $\mathrm{S} \leftrightarrow . \mathrm{S}(\mathrm{X} \leftrightarrow \mathrm{Y})=1 \Leftrightarrow \mathrm{S}(\mathrm{X})=\mathrm{S}(\mathrm{Y})$.

- $\quad S \neg$ nat. $S(\neg X)=1 \Leftrightarrow S(X)=0$ donde $X$ es una fórmula no atómica.

- $\quad \mathrm{SI} . \mathrm{S}(\mathrm{Ip})=0 \Leftrightarrow \mathrm{S}(\mathrm{p})=\mathrm{S}(\neg \mathrm{p})=1$ donde $\mathrm{p}$ es una fórmula atómica.

- $\quad$ Se dice que una fórmula $X$ es válida si y solo si para toda sociedad $s, S(X)=1$.

\section{Caracterización de los árboles de forzamiento}

Se define la Complejidad c como una función que asigna a cada fórmula de LSA un entero no negativo de la siguiente forma:

- $\quad C(q)=0$, donde q es una fórmula cuasiatómica.

- $\quad C(I p)=1$, donde $p$ es una fórmula atómica.

- $C(X k Y)=1+$ máximo de $\{C(X), C(Y)\}$, donde $k \in\{\wedge, \vee, \rightarrow$, $\leftrightarrow\}$.

- $C(\sim X)=1+C(X)$.

- $\quad C(\neg Y)=1+C(Y)$, donde $Y$ es una fórmula no atómica.

Se define la Profundidad $\mathrm{P}$ como una función que asigna a cada nodo de un árbol inicial un entero no negativo de la siguiente forma:

- $\quad P(q)=0$, donde q es una fórmula cuasi-atómica.

- $\quad P(I P)=1$, donde $p$ es una fórmula atómica.

- $\mathrm{P}(\operatorname{Ar}[\mathrm{XkY}])=1$ + máximo de $\{\mathrm{P}(\operatorname{Ar}[\mathrm{X}]), \mathrm{P}(\operatorname{Ar}[\mathrm{Y}])\}$, donde $k \in\{\Lambda, \vee, \rightarrow, \leftrightarrow\}$.

- $P(\operatorname{Ar}[\sim X])=1+P(\operatorname{Ar}[X])$.

- $\quad \mathrm{P}(\operatorname{Ar}[\neg \mathrm{Y}])=1+\mathrm{P}(\operatorname{Ar}[\mathrm{Y}])$, donde $\mathrm{Y}$ es una fórmula no atómica.

Proposición. Sociedad abierta correspondiente a una función de marca

Para cada fórmula $X$ de LSA y cada función de marca $m$, existe una sociedad $s_{m}$, tal que, $M(R[X])=1 \Leftrightarrow S_{m}(X)=1$.

Prueba: Sea X una fórmula de LSA, y sea $m$ una función de marcas para $X$. Se define la función $s_{m}$ del conjunto de fórmulas cuasiatómicas en el conjunto $\{0,1\}$ de la siguiente manera:

Si $m_{q}$ es una marquilla de $m$ entonces $\mathrm{Ag}_{q}$ es un agente de $s_{m}$, donde $\operatorname{Ag}_{q}(p)=m_{q}(p)$.

La función $S_{m}$ se extiende a una función $S_{m}$ del conjunto de fórmulas de $L C$ en el conjunto $\{0,1\}$, mediante las reglas primitivas para las sociedades de la sección anterior. Se tiene entonces que $S_{m}$ es una sociedad de LSA.

A fin de probar que $M(R[X])=1 \Leftrightarrow S_{m}(X)=1$, se procede por inducción sobre la complejidad de la fórmula $X$.

Paso base: supóngase que la $C(X)=0$, esto significa que $X$ es una fórmula cuasiatómica.

- $\quad$ Caso 1: $X=p$, con $p$ atómica. $m(p)=1 \Leftrightarrow \exists m q \in m, m q(p)$ $=1$, por la definición de sm resulta $m(p)=1 \Leftrightarrow \exists A g q \in s m$, $\operatorname{Agq}(p)=1$, por la regla sat se obtiene $m(p)=1 \Leftrightarrow S m(p)=1$, es decir $m(p)=s m(p)$. 
- Caso 2: $X=\neg p$, con $p$ atómica. $\mathrm{m}(\neg \mathrm{p})=1 \Leftrightarrow \exists \mathrm{mq} \in \mathrm{m}$, $\mathrm{mq}(\mathrm{p})=0$, por la definición de sm resulta $\mathrm{m}(\neg \mathrm{p})=1 \Leftrightarrow$ $\exists \mathrm{Ag} q \in \mathrm{sm}, \mathrm{Agq}(\mathrm{p})=0$, por la regla $\mathrm{s} \neg$ at se obtiene $\mathrm{m}(\neg \mathrm{p})$ $=1 \Leftrightarrow \operatorname{Sm}(\neg p)=1$, es decir $\mathrm{m}(\neg \mathrm{p})=\operatorname{sm}(\neg \mathrm{p})$.

Se tiene entonces que $m(X)=s_{m}(X)$, donde $X$ es cuasiatómica. En consecuencia, se tienen, $R[X]=X, M(X)=$ $m(X), s_{m}(X)=m(X)$ y $S_{m}(X)=s_{m}(X)$.

Se concluye entonces que $M(R[X])=1 \Leftrightarrow S_{m}(X)=1$.

Paso de inducción: Supóngase que $C(X) \geq 1$.

Al ser $C(X) \geq 1, X$ debe ser una fórmula no cuasiatómica, es decir, $X$ tiene una de las siguientes formas: Ip, $\neg \mathrm{W}$ (W no atómica), $Y \wedge Z, Y \vee Z, Y \rightarrow Z, Y \leftrightarrow Z, \sim Y$ (todos los casos, excepto el primero y el segundo, se prueban como en Sierra (2006)). Se analizan los casos 1 y 2.

- $\quad$ Caso 1: sea $X=I p$, donde $p$ es atómica. Se tiene que $R[X]$ $=I$, por lo que $M(R[X])=0 \Leftrightarrow M(I)=0$, pero por la regla Rlat se tiene $M(I)=0 \Leftrightarrow[M(p)=M(\neg p)=1]$, lo cual por las reglas Aat y $A \neg$ at implica $M(I)=0 \Leftrightarrow[\exists m 1 \in m, m 1(p)=1$ y $\exists \mathrm{m} 2 \in \mathrm{m}, \mathrm{m} 2(\mathrm{p})=0]$. En consecuencia, se tiene que $\mathrm{M}(\mathrm{I})=$ $0 \Leftrightarrow\left[\exists A_{1} \in s m, A g 1(p)=1\right.$ y $\left.\exists A_{2} \in s m, A g 2(p)=0\right]$, por las reglas sat y $s \neg$ at se infiere $M(I)=0 \Leftrightarrow[\mathrm{sm}(\mathrm{p})=\operatorname{sm}(\neg \mathrm{p})=$ 1], lo cual por la regla SI significa $M(I)=0 \Leftrightarrow[S m(I P)=0]$, es decir, $M(R[X])=1 \Leftrightarrow \operatorname{Sm}(X)=1$.

- Caso 2: sea $X=\neg W$ con $W$ no atómica. Se tiene que $R[X]=$ $\neg$, por lo que $M(R[X])=1 \Leftrightarrow M(\neg)=1$, pero por $A \neg$ nat se tiene $M(\neg)=1 \Leftrightarrow M(a \neg)=0$, y como además $a \neg=R[W]$, resulta que $M(R[X])=1 \Leftrightarrow[M(R[W])=0]$. Al utilizar la hipótesis inductiva se tiene que $M(R[X])=1 \Leftrightarrow S_{m}(W)=0$. Por la definición de $S m \neg$ nat se concluye que $M(R[X])=1$ $\Leftrightarrow S_{m}(\neg W)=1$, es decir, $M(R[X])=1 \Leftrightarrow S_{m}(X)=1$.

Se tiene entonces que para todos los casos $M(R[X])=1$ $\Leftrightarrow S_{m}(X)=1$, de modo que queda así probado el paso de inducción. Por el principio de Inducción se concluye que: $M(R[X])=1 \Leftrightarrow S_{m}(X)=1$.

Se ha probado entonces que para cada fórmula X de LSA y para cada función de marca $m$, existe una sociedad $s_{m}$, tal que, $M(R[X])=1 \Leftrightarrow S_{m}(X)=1$.

\section{Proposición. Función de marca asociada a una sociedad abierta}

Para cada fórmula $X$ de LSA y para cada sociedad abierta $s$, existe una función de marca $m_{s}$, tal que, $M_{s}(R[X])=1 \Leftrightarrow S(X)=1$.

Prueba: sea $X$ una fórmula de LSA y sea $s$ una sociedad abierta. Se define la función de marca $m_{s}$ del conjunto de hojas del árbol de $\mathrm{X}$ en el conjunto $\{0,1\}$ de la siguiente forma:

$\mathrm{Si} \mathrm{Ag}_{q}$ es un agente de $s$ entonces $\mathrm{m}_{q}$ es una marquilla de $m_{s}$, donde $m_{q}(p)=\operatorname{Ag}_{q}(p)$.

La función $m_{s}$ se extiende a una función $M_{s}$ del conjunto de nodos del árbol de $X$ en el conjunto $\{0,1\}$, mediante las reglas primitivas para el forzamiento de marcas presentadas en la sección “Marcando los nodos de un árbol”. Se tiene entonces que $m_{s}$ es una función de marca de nodos.
Para probar que $M_{s}(R[X])=1 \Leftrightarrow S(X)=1$, se procede por inducción sobre la profundidad de $\operatorname{Ar}[\mathrm{X}]$.

Paso base: supóngase que $\mathrm{P}(\operatorname{Ar}[\mathrm{X}])=0$, esto significa que $X$ es una fórmula cuasiatómica.

- $\quad$ Caso 1: $X=p$, con $p$ atómica. $s(p)=1 \Leftrightarrow \exists A g q \in s, A g q(p)$ $=1$, por la definición de $\mathrm{ms}$ resulta $\mathrm{s}(\mathrm{p})=1 \Leftrightarrow \exists \mathrm{mq} \in \mathrm{ms}$, $m q(p)=1$, por la regla Aat se obtiene $s(p)=1 \Leftrightarrow m s(p)=1$, es decir $s(p)=m s(p)$.

- $\quad$ Caso 2: $X=\neg p$, con $p$ atómica. $s(\neg p)=1 \Leftrightarrow \exists$ Agq $\in s$, $\operatorname{Agq}(p)=0$, por la definición de ms resulta $s(\neg p)=1 \Leftrightarrow$ $\exists \mathrm{mq} \in \mathrm{ms}, \mathrm{mq}(\mathrm{p})=0$, por la regla $\mathrm{A} \neg$ at se obtiene $\mathrm{s}(\neg \mathrm{p})=$ $1 \Leftrightarrow \operatorname{ms}(\neg p)=1$, es decir, $s(\neg p)=m s(\neg p)$.

Se tiene entonces que $s(X)=m_{s}(X)$, donde $X$ es cuasi-atómica. En consecuencia, se tienen, $R[X] \stackrel{s}{=} X, M_{s}(X)=m_{s}(X), m_{s}(X)=s(X)$ y $s(X)=S(X)$. Se concluye entonces que $M_{s}(R[X])=1 \Leftrightarrow S(X)=1$.

Paso de inducción: supóngase que $P(\operatorname{Ar}[X]) \geq 1$.

Al ser $P(\operatorname{Ar}[X]) \geq 1, X$ debe ser una fórmula no cuasiatómica, es decir, $X$ tiene una de las siguientes formas: I $p, \neg \mathrm{W}$ (con W no atómica), $Y \wedge Z, Y \vee Z, Y \rightarrow Z, Y \leftrightarrow Z, \sim Y$ (todos los casos, excepto el primero y el segundo, se prueban como en Sierra (2006)). Se analizan los casos 1 y 2 .

Caso 1: Sea $X=I p$, donde $p$ es atómica. Se tiene que $R[X]$ $=\mathrm{I}$, por lo que $\mathrm{Ms}(\mathrm{R}[\mathrm{X}])=0 \Leftrightarrow \mathrm{Ms}(\mathrm{I})=0$, pero por la regla Rlat se tiene $M s(I)=0 \Leftrightarrow[M s(p)=M s(\neg p)=1]$, lo cual por las reglas Aat y $A \neg$ at implica $M s(I)=0 \Leftrightarrow[\exists m 1 \in m s, m 1(p)=1$ y $\exists \mathrm{m} 2 \in \mathrm{ms}, \mathrm{m} 2(\mathrm{p})=0]$. En consecuencia, se tiene que $\mathrm{Ms}(\mathrm{I})=$ $0 \Leftrightarrow\left[\exists \mathrm{Ag}_{1} \in \mathrm{s}, \mathrm{Ag}_{1}(\mathrm{p})=1\right.$ y $\left.\exists \mathrm{Ag}_{2} \in \mathrm{s}, \mathrm{Ag}_{2}(\mathrm{p})=0\right]$, por las reglas sat y $s \neg$ at se infiere $M s(I)=0 \Leftrightarrow[S(p)=S(\neg p)=1]$, lo cual por la regla SI significa $\mathrm{Ms}(\mathrm{I})=0 \Leftrightarrow[S(I \mathrm{P})=0]$, es decir, $\mathrm{Ms}(\mathrm{R}[\mathrm{X}])$ $=1 \Leftrightarrow S(X)=1$.

- $\quad$ Caso 2: Sea $X=\neg W$, con W no atómica. Se tiene que $R[X]$ $=\neg$, por lo que $M s(R[X])=1 \Leftrightarrow M s(\neg)=1$, pero por $A \neg$ nat se tiene $\operatorname{Ms}(\neg)=1 \Leftrightarrow \operatorname{Ms}(a \neg)=0$, y como además $a \neg=$ $\mathrm{R}[\mathrm{W}]$, resulta que $\mathrm{Ms}(\mathrm{R}[\mathrm{X}])=1 \Leftrightarrow \mathrm{Ms}(\mathrm{R}[\mathrm{W}])=0$. Utilizando la hipótesis inductiva se tiene que $\mathrm{Ms}(\mathrm{R}[\mathrm{X}])=1 \Leftrightarrow \mathrm{S}(\mathrm{W})=$ o. Por la definición de $S \neg$ nat se concluye que $\operatorname{Ms}(R[X])=1$ $\Leftrightarrow S(\neg W)=1$, es decir, $M s(R[X])=1 \Leftrightarrow S(X)=1$.

Se tiene entonces que para todos los casos $M_{s}(R[X])=1 \Leftrightarrow$ $S(X)=1$, de modo que queda así probado el paso de inducción. Por el principio de inducción se concluye que: $M_{s}(R[X])=1 \Leftrightarrow S(X)=1$.

Se ha probado, entonces, que para cada fórmula X de LSA y para cada sociedad abierta $s$, existe una función de marca $m_{s}$, tal que, $M_{s}(R[X])=1 \Leftrightarrow S(X)=1$.

Proposición. Caracterización semántico-deductiva de los árboles de forzamiento

1. La fórmula $X$ es válida desde el punto de vista de los árboles si y solo si $\mathrm{X}$ es válida desde el punto de vista de las sociedades.

2. 2. La fórmula $X$ es válida desde el punto de vista de los árboles si y solo si $\mathrm{X}$ es un teorema del sistema deductivo paraconsistente $\mathrm{P}^{1}$. 
Prueba: supóngase que $X$ no es válida desde el punto de vista de los árboles. En consecuencia, se tiene que existe una función de marca $m$, tal que $M(R[X])=0$. Se tiene, entonces, por la proposición inicial de esta sección, que existe una sociedad abierta $s_{m}$, tal que $S_{m}(X)=0$, por tanto, $X$ no puede ser válida desde el punto de vista de las sociedades abiertas.

Supóngase ahora que $X$ no es válida desde el punto de vista de las sociedades abiertas, entonces, existe una sociedad $s$, tal que $S(X)=0$. Se tiene, entonces, por la proposición previa, que existe una función de marca $m_{s}$, tal que $M_{s}(\mathrm{R}[\mathrm{X}])=0$, por tanto, $\mathrm{X}$ no puede ser válida desde el punto de vista de los árboles.

Se concluye así que $X$ es válida desde el punto de vista de los árboles si y solo si $\mathrm{X}$ es válida desde el punto de vista de las sociedades abiertas.

De acuerdo con Carnielli y Lima-Marques (1999) se sabe que los teoremas de $\mathrm{P}^{1}$ son exactamente las fórmulas válidas desde el punto de vista de las sociedades abiertas; así, entonces, como consecuencia de la parte 1 , se tiene que la fórmula $X$ es válida desde el punto de vista de los árboles si y solo si $X$ es un teorema del sistema deductivo $\mathrm{P}^{1}$.

\section{Ilustraciones}

Un nodo encerrado en un círculo indica que el nodo está marcado con 1 por la función de marca, un nodo encerrado en un cuadro indica que el nodo está marcado con o por la función de marca (de esta forma se presenta en Sierra (2001; 2006).
Un nodo encerrado en un doble círculo indica que el nodo está marcado con 1 por la marquilla(s) indicada(s) en la rama que lo precede, un nodo encerrado en un doble cuadro indica que el nodo está marcado con o por la marquilla(s) indicada(s) en la rama que lo precede.

Se sabe que una fórmula es A-válida si la raíz del árbol está forzosamente marcada con 1, RM1. Por otra parte, un árbol de forzamiento está mal marcado cuando su raíz se encuentra marcada con 0 , y además existen dos nodos asociados a una misma fórmula, los cuales tienen marcas contrarias. Lo anterior significa que cuando el árbol de una fórmula está mal marcado, entonces la fórmula es A-válida.

Un árbol de forzamiento está bien marcado cuando su raíz está marcada con 0 , todos los nodos están marcados respetando las reglas y no existen dos nodos asociados a una misma fórmula, los cuales tienen marcas contrarias. Lo anterior significa que cuando el árbol de una fórmula está bien marcado, entonces la fórmula no es A-válida. Además, las marquillas de los nodos que se derivan de las hojas del árbol inicial determinan la semántica de sociedades que refuta la validez de la fórmula.

\section{Ilustración 1}

En la (Fig. 3) se muestra un árbol de forzamiento mal marcado para la fórmula $A$-válida $I A \rightarrow[\neg A \rightarrow(A \rightarrow B)]$, donde $A$ y $B$ son fórmulas atómicas, así como el árbol de forzamiento bien marcado de la fórmula $A$-inválida $\neg A \rightarrow(A \rightarrow B)$.

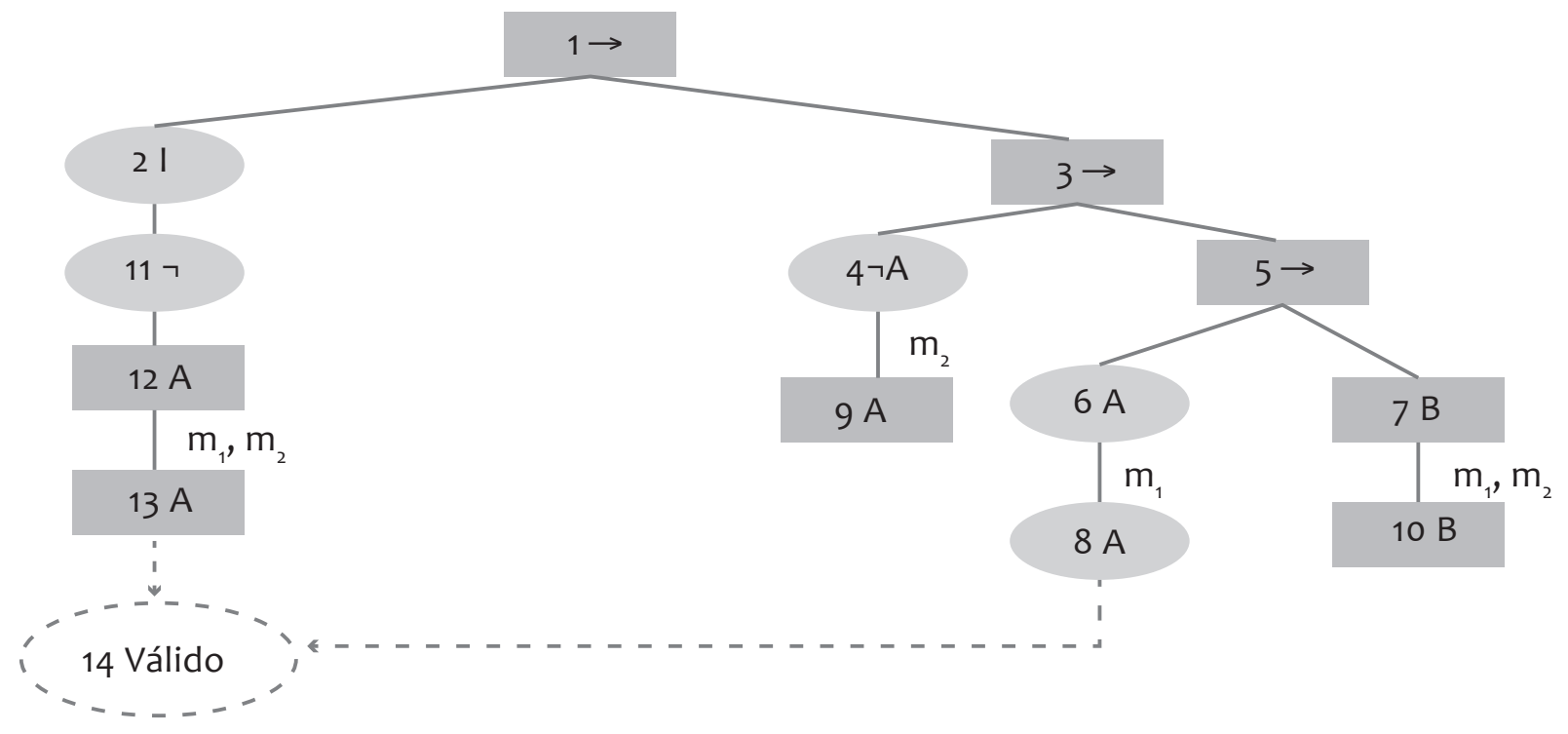

Justificaciones:

Figura 3
1. $R R$
9. $A \neg$ at en 4
2, 3. $\mathrm{R} \rightarrow$ en 1
4, 5. $\mathrm{R} \rightarrow$ en 3
6, 7. $R \rightarrow$ en 5
8. Aat en 6
11. IA en 4
12. A $\neg$ Alat en 11 y 2
13. Rat en 12

14. DM en 8 y 13

(o también DM en 12 y 6) 
Obsérvese, además, que los pasos del 3 al 10 determinan un árbol bien marcado para la fórmula $\neg A \rightarrow(A \rightarrow B)$, y, en consecuencia, esta es A-inválida. Además, las marquillas indican que la fórmula es refutada por la siguiente sociedad abierta: $s=$ $\left\{\mathrm{Ag}_{1}, \mathrm{Ag}_{2}\right\}$, tal que $A g_{1}(\mathrm{~A})=1$ y $\mathrm{Ag}_{2}(\mathrm{~A})=\mathrm{Ag}_{1}(\mathrm{~B})=\mathrm{Ag}_{2}(\mathrm{~B})=0$.

\section{Ilustración 2}

En la (Fig. 4) se muestra un árbol de forzamiento mal marcado para la fórmula A-válida IB $\rightarrow\{(A \rightarrow B) \rightarrow[(A \rightarrow \neg B)$ $\rightarrow \neg A]\}$, donde $A$ y $B$ son fórmulas atómicas, así como el árbol de forzamiento bien marcado de la fórmula A-inválida $(A \rightarrow B)$ $\rightarrow[(\mathrm{A} \rightarrow \neg \mathrm{B}) \rightarrow \neg \mathrm{A}]$.

Obsérvese, además, que los pasos del 3 al 15 determinan un árbol bien marcado para la fórmula $(A \rightarrow B) \rightarrow[(A \rightarrow \neg B)$ $\rightarrow \neg A]$, y, en consecuencia, esta es $A$-inválida. Adicionalmente, las marquillas indican que la fórmula es refutada por la siguiente sociedad abierta: $s=\left\{\mathrm{Ag}_{1}, \mathrm{Ag}_{2}, \mathrm{Ag}_{3}\right\}$, tal que $A g_{1}(\mathrm{~A})=$ $A g_{2}(A)=A g_{3}(A)=1, \operatorname{Ag}_{2}(B)=1$ y $A g_{3}(B)=0$.

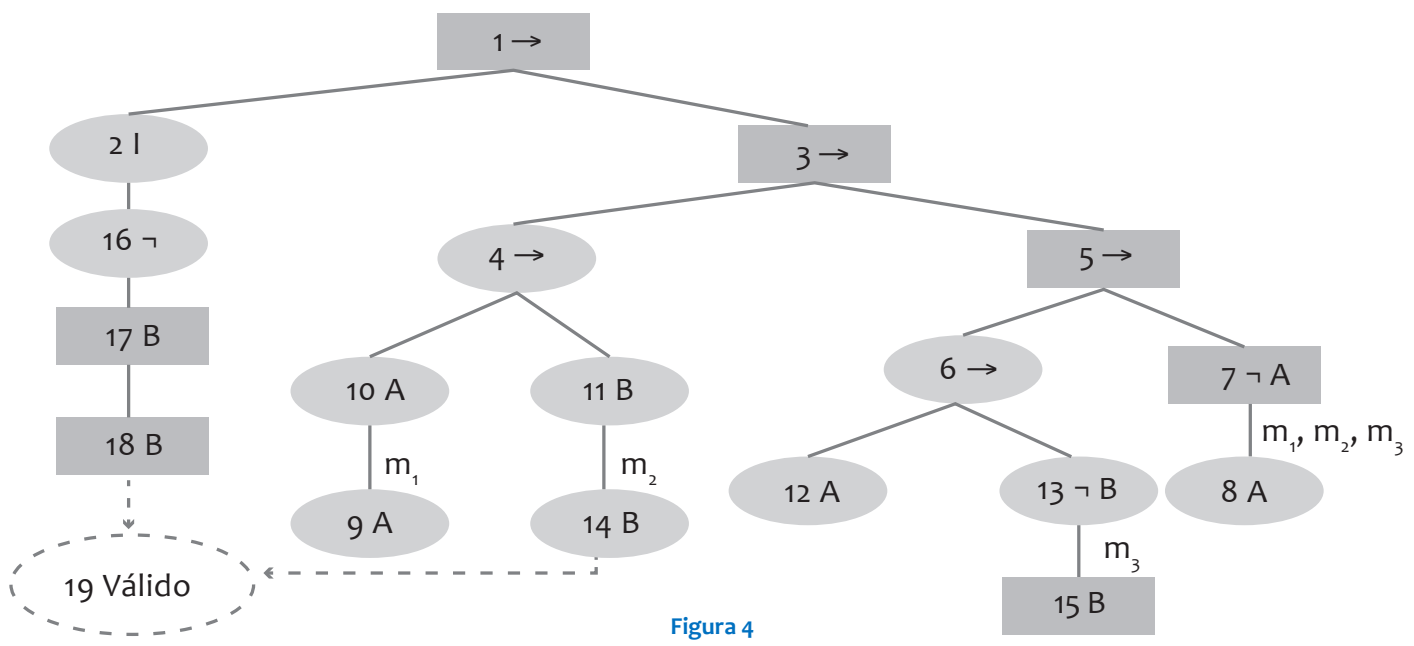

Justificaciones:
1. $R R$
2, 3. $\mathrm{R} \rightarrow$ en 1
4, 5. $R \rightarrow$ en 3
6, 7. $\mathrm{R} \rightarrow$ en 5
8. $R \neg$ at en 7
9. IA en 8
10. Aat en 9
11. $\mathrm{Ai} A \rightarrow$ en 10 y 4
12. IA en 10
13. AiA $\rightarrow$ en 12 y 6
14. Aat en 11
16. IA en 13
17. A Alat en 16 y 2
18. Rat en 17

15. $A \neg$ at en 13

19. $\mathrm{DM}$ en 18 y 14 (o

también DM en 17 y 11)

El análisis que se realizó a la fórmula IB $\rightarrow\{(A \rightarrow B) \rightarrow[(A$ $\rightarrow \neg B) \rightarrow \neg A]\}$, corresponde a la formalización del siguiente argumento: si en un grupo de individuos, una propuesta genera consecuencias que son aceptadas por algunos individuos $(A \rightarrow B)$ y rechazadas por otros individuos $(A \rightarrow \neg B)$, entonces no se puede asegurar que la propuesta sea rechazada por algunos individuos $(\neg A)$, salvo que exista consenso entre los individuos del grupo con respecto a la aceptación o el rechazo de las consecuencias (IB).

\section{Ilustración 3}

En la (Fig. 5) se muestra un árbol de forzamiento con la raíz forzosamente marcada con 1 para la fórmula $A$-válida $\neg(A \wedge B)$ $\rightarrow(\neg A \vee \neg B)$, donde $A$ y $B$ son fórmulas atómicas. El análisis realizado a la fórmula $\neg(A \wedge B) \rightarrow(\neg A \vee \neg B)$ corresponde a la formalización del siguiente argumento: si un grupo de individuos rechaza la ejecución simultánea de dos proyectos $(\neg(A \wedge B))$, entonces la ejecución de uno de los proyectos es rechazada por algunos individuos $(\neg A \vee \neg B)$.

\section{Ilustración 4}

En la (Fig. 6) se muestra un árbol de forzamiento mal marcado para la fórmula A-válida $(I A \wedge I) \rightarrow[(\neg A \vee \neg B) \rightarrow \neg(A \wedge$ $B)$ ], donde $A$ y $B$ son fórmulas atómicas, así como el árbol de forzamiento bien marcado de la fórmula $A$-inválida $(\neg A \vee \neg B)$ $\rightarrow \neg(A \wedge B)$.

Obsérvese, además, que los pasos del 3 al 10, junto con los del 15 al 18 (al justificar 15 como OR), determinan un árbol bien marcado para la fórmula $(\neg A \vee \neg B) \rightarrow \neg(A \wedge B)$, y en consecuencia, esta es A-inválida. Adicionalmnte, las marquillas indican que la fórmula es refutada por la siguiente sociedad abierta: $s=\left\{\mathrm{Ag}_{1}, \mathrm{Ag}_{2}, \mathrm{Ag}_{3}\right\}$, tal que $\mathrm{Ag}_{1}(\mathrm{~A})=\mathrm{Ag}_{2}(\mathrm{~A})=\mathrm{Ag}_{3}(\mathrm{~A})=1$, $\mathrm{Ag}_{2}(\mathrm{~B})=1$ y $\mathrm{Ag}_{3}(\mathrm{~B})=0$.

El análisis que se realizó a la fórmula $(I A \wedge I B) \rightarrow[(\neg A \vee$ $\neg B) \rightarrow \neg(A \wedge B)]$ corresponde a la formalización del siguiente argumento: si en un grupo de individuos, algunos de ellos 
rechazan la compra de uno de los productos $(\neg A \vee \neg B)$, entonces no se puede asegurar que el grupo rechace comprar ambos productos $(\neg(A \wedge B))$, salvo que haya consenso entre los individuos del grupo con respecto a la compra o no de cada uno de los productos $(\mathrm{I} A \wedge \mathrm{IB})$.

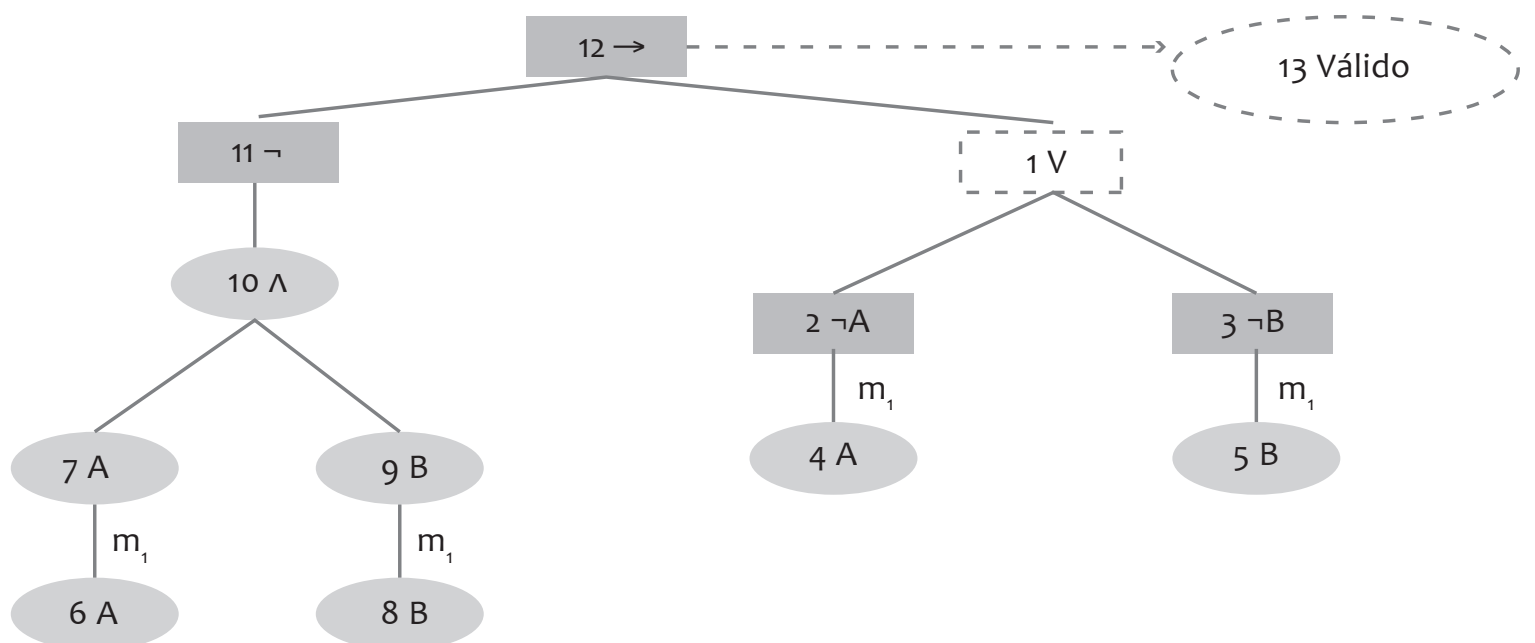

Figura 5

Justificaciones:
1. $\mathrm{OR}$
2, 3. $R v$ en 1
4. $\mathrm{R} \neg$ at en 2
5. $R \neg$ at en 3
6. IA en 4
7. Aat en 6
8. IA en 5
9. Aat en 8
10. AiAd^ en 7 y 9
11. Aa $\neg$ nat en 10

12. ORd-Ri $\rightarrow$ en 1,1113 .

RM1 en 12

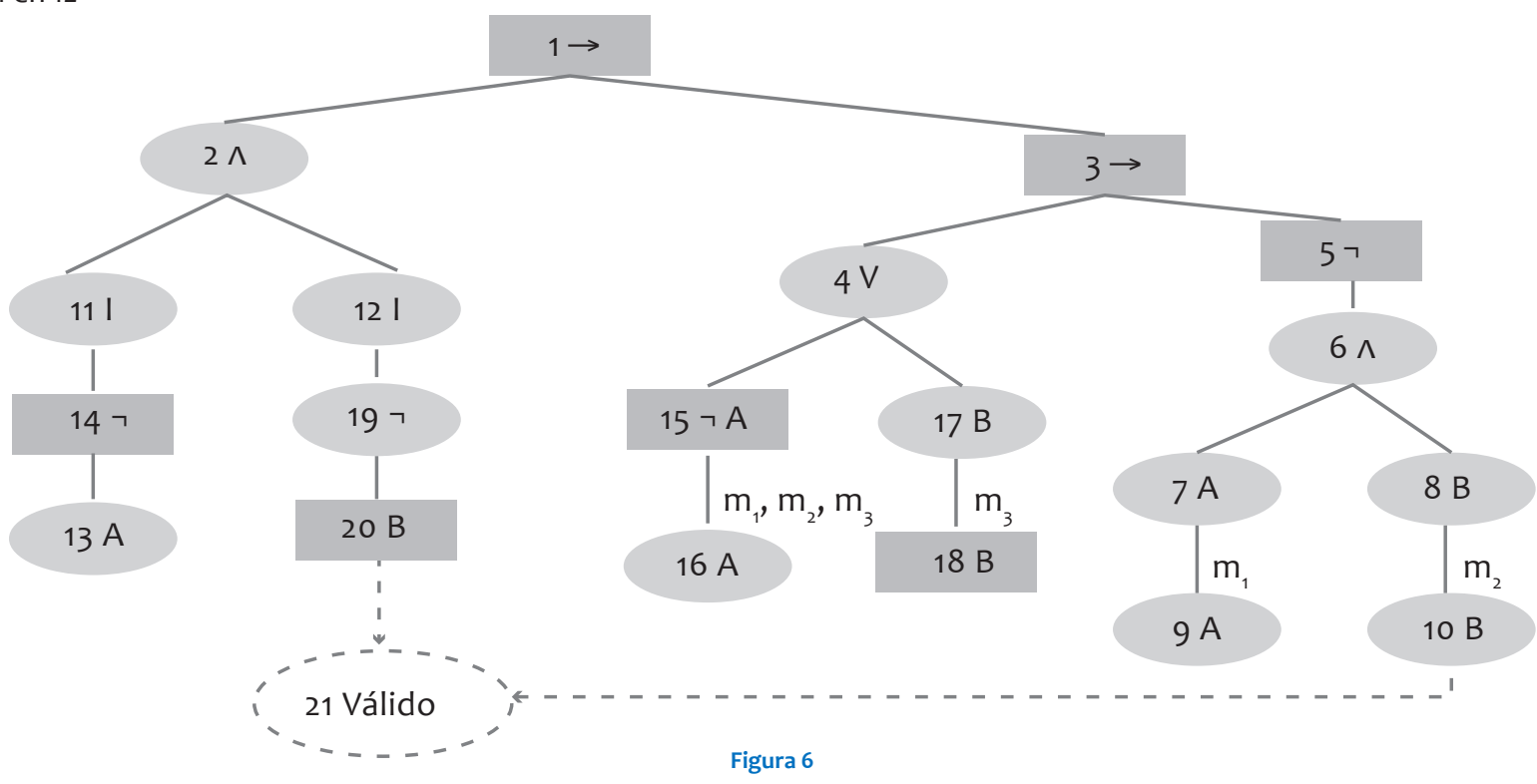

Justificaciones:
1. $R R$
2, 3. $\mathrm{R} \rightarrow$ en 1
4, 5. $\mathrm{R} \rightarrow$ en 3
6. $R \neg$ nat en 5
7, 8. A^ en 6
9. Aat en 7
10. Aat en 8
11, 12. A^ en 2
13. IA en 7

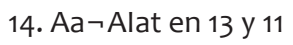
15. IR en 14
16. $R \neg$ at en 1517. RiAv en 15 y 4
18. $A \neg$ at en 17
19. IA en 17
20. AᄀAlat en 19 y 12
21. DM en 20 y 8 


\section{CONCLUSIONES}

Con los árboles de forzamiento semántico para sociedades abiertas, la validez de una fórmula de LSA se puede determinar de manera visual y completamente mecánica. Así, por ejemplo, mediante un algoritmo se recorre el árbol de la fórmula al buscar en cada nodo la aplicación de una regla para marcarlo. Cuando la fórmula es inválida, es decir, cuando el árbol de la fórmula está bien marcado, entonces la lectura de las marquillas asociadas a las fórmulas cuasiatómicas proporciona los agentes de una sociedad abierta, con la cual se refuta la validez de la fórmula.

Desde el punto de vista didáctico, la simplicidad de las reglas para el forzamiento de marcas, en comparación con las deducciones de los sistemas axiomáticos P1 y LBPcAt, hacen de los árboles de forzamiento para sociedades abiertas una herramienta de trabajo muy útil.

\section{REFERENCIAS}

- Barrero, T. y Carnielli, W. (2005). Tableaux sin refutación. Matemáticas: Enseñanza Universitaria, 13(2), 81-99.

- Beth, E. (1962). Formal Methods, an Introduction to Symbolic Logic and to the Study of Effective Operations in Arithmetic and Logic. Dordrecht: Reidel Publishing.

- Carnielli, W. (1987). Systematization of Finite Many-Valued Logics Through the Method of Tableaux. The Journal of Symbolic Logic, 52(2), 473-493.

- Carnielli, W. y Lima Marquez, M. (1999). Society Semantics and MultipleValued Logics. Contemporary Mathematics, 235, 3-52. Recuperado de https:// goo.gl/ig3bto.

- Fitting, M. (1971). A Tableau Proof Method Admitting the Empty Domain. Notre Dame Journal of Formal Logic, 12(2), 219-224.

- Guarín, H. y Montoya, M. (2003). Semántica de sociedades como un modelo o interpretación de las lógicas básicas paraconsistente y paracompleta a nivel atómico (Trabajo de grado). Especialización en Lógica y Filosofía, Universidad Eafit.

- Sette, A. (1973). On the Propositional Calculus P1. Mathematic Japonicae, 18(13), 173-180.

- Sierra, M. (2001). Árboles de forzamiento semántico. Revista Universidad Eafit, 37(123), 53-72.

- Sierra, M. (2003). Inferencia visual para la lógica básica paraconsistente y paracompleta. Medellín: MS-Print.

- Sierra, M. (2006). Caracterización deductiva de los árboles de forzamiento semántico. Revista Ingeniería y Ciencia, 2(3), 73-102. Recuperado de https:// goo.gl/rarWPW.

- Smullyan, R. (1968). First Order Logic. Berlín: Springer-Verlag. 